\title{
Ueber die wahrseheinliche Lebensdauer der assyrisch-babylonischen Sprache.
}

\author{
Von G. F. F. Gutbrod.
}

EberiaARd Schrader schrieb im Jahr 1869: „Nichts hindert anzunehmen, dass sofort nach dem Sturze des Chaldäerreiches wie aramäische Bevölkerung, so aramäische Sprache in das chaldäische Gebiet eindrang; dass wie später sogar in Palästina, so auch in Babylonien das Aramäische seit dieser Zeit allmählich die Volkssprache ward, wie denn eben diese Sprache schon früher die allgemeine Verkehrssprache in den Ländern zwischen dem Tigris und dem Mittelmeere bildete ". ${ }^{\text {) }}$ )

Noch weiter geht Th. Nöldeke: „Die einheimische Keilschrift wurde in Babylonien nicht bloss noch in der Perserzeit gebraucht, sondern man hat dort sogar Urkunden mit solcher aus der griechischen Periode aufgefunden. Natürlich folgt daraus nicht, dass damals noch assyrisch gesprochen wurde. Ja es ist möglich, dass diese Sprache schon vor dem Untergange Ninives aus dem Leben verschwunden und nur noch officielle und Priestersprache war. Die Inschriften waren doch jedenfalls immer nur für einen kleinen Kreis von Schriftgelehrten bestimmt. Auch die zahlreichen babylonischen Privatkontrakte auf Thontäfelchen konnten sicher von den wenigsten der Kon-

1) Zeitschr. der D. M. G. 23, S. 37 I. Vgl. ạuch KGF. S. 64 ff. 
trahierenden selbst gelesen werden, brauchen daher durchaus nicht in deren lebender Sprache verfasst zu sein. ${ }^{~}{ }^{z}$ )

Ueber blosse Vermutungen kommt man natürlich in dieser Frage nicht hinaus, doch scheint uns NöLdEkE mit der Toterklärung der assyrisch-babylonischen Sprache gar zu sehr zu eilen. Den Untergang Niniveh's und Babylons wird sie doch wohl noch erlebt haben, auch als gesprochene Sprache. Im Sommer 1887 sah Verf. dieses einen in Babylonien (wo? ist mir nicht näher bekannt geworden) gefundenen, damals im Besitz des Rev. J. Hechler befindlichen Backstein mit bilinguer Inschrift, die in aramäischer (der Quadratschrift sehr ähnlicher) und griechiהרדנרנאח scher Schrift nichts weiter als den Personennamen

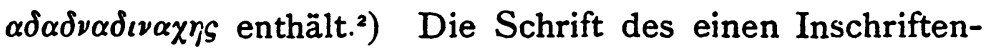
teils und das erste Namenselement sind entschieden aramäisch, die Sprache des zweiten und dritten Namenselements ist aber ebenso entschieden assyrisch. Wenn man nun bedenkt, welch grossen Einfluss auf die Namenschöpfung gerade die lebende Volkssprache auszuüben pflegt, so wird es nicht allzu gewagt erscheinen, wenn ich diese, leider undatierte, Ziegelinschrift als einen Beweis dafür geltend machen möchte, dass noch weit in die hellenistische Zeit herein die alte babylonisch-assyrische Sprache, wenn auch mit aramäischen Elementen vermischt, im Munde des Volkes fortlebte.

Darüber kann sicherlich kein Zweifel obwalten, dass diese Sprache als hieratische und Gelehriensprache noch

1) Die semitischen Sprachen S. $4 \mathrm{I}$ f.

2) Leider war es dem Verf. nicht möglich, einen Abklatsch von der Inschrift zu nehmen; wo der Backstein sich jetzt befindet, hat er auch nicht in Erfahrung bringen können. [Der Verf. meint die Backsteine von Tello, von welchen sich u. A. Exx. in den Museen von Paris und Berlin befinden. Die Inschrift wurde von DE VoGüÉ, SCHRADER, sowie im Corpus inscriptionum semiticarum, unter Beigabe von graphischen Nachbildungen, behandelt (s. die Literatur bei Schrader, Keilinschr. Bibliothek III, 2 S. 142, Note I). Die Inschrift wird gemäss dem Typus der aramäischen Schriftzeichen von EUting in die Zeit 310-250 v. Chr. gesetzt. - Red.] 
weit länger sich behauptete. StrassmaIer hat in dieser Zeitschrift (III, S. 129 ff.) eine Reihe von Keilschrifttexten veröffentlicht, welche bis ins Jahr 80 v. Chr. herabreichen. ${ }^{x}$ ) Darunter sind ein paar Texte astronomischen und astrologischen Inhalts, und Verf. dieses möchte den Satz aufstellen, dass die assyrisch-babylonische Sprache samt der Keilschrift ebenso lange ihr Dasein fristete, als die babylonischen Geheimwissenschaften, Magie und Astrologie, natürlich eben in den „kleinen Kreisen der Schriftgelehrten“.

Diese Wissenschaften blieben freilich, wenn auch die Namen Chaldaei oder Babylonii den Adepten derselben fortwährend anhafteten, keineswegs ein Monopol der babylonischen Schriftgelehrten; hat doch schon Cicero (de divin. I, I, 2) für nötig gefunden, zu konstatieren, dass die Chaldaei non ex artis, sed ex gentis vocabulo so benannt seien, und je weiter in die Kaiserzeit herein, desto zutreffender wird die Bemerkung JAKOB BuRcKhardT's ${ }^{2}$ ) sein, dass diese sog. Chaldäer ,nur geringsten Teils wirklich aus dem Lande am untern Euphrat stammen mochten". Doch hat immerhin neben den übrigen Ländern des Orients, aus welchem nach FRIEDLÄNDER ${ }^{3}$ ) und J. RÉvILLE ${ }^{4}$ ) die meisten Astrologen und Magier der Kaiserzeit stammten, gewiss auch das alte Chaldäa noch in dieser Zeit sein nicht zu verachtendes Kontingent gestellt. Juvenal z. B. (unter

I) Vgl. Strassmaier, a. a. O., S. 135; auch Oppert, in dieser Zeitschrift IV, p. 399. [Pater STrassmaIER ermächtigt mich mitzuteilen, dass die Inschrift $8 \mathrm{I}, 7-6,2 \mathrm{I}$, die er jüngst copirt hat, das folgende Datum

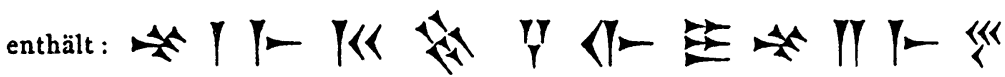

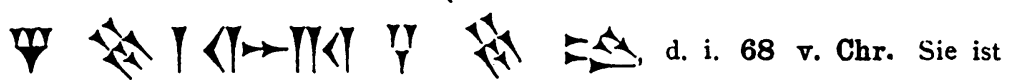
somit die jüngste der bis jetzt bekannten datirten babylonischen Keilininschriften. - Bezold].

2) Die Zeit Konstantins des Grossen ${ }^{2}$ S. 211.

3) Darstellungen aus der Sittengeschichte Roms I5, S. I15. 324.

4) Die Religion zu Rom unter den Severern, deutsch von G. KRÜGER S. 135 . 
Domitian) führt Sat. 6, $553 \mathrm{f}$. neben den kleinasiatischen Bellonarii und Galli (v. 5 I I ff.), den ägyptischen Isispriestern (v. $522 \mathrm{ff}$ ), den wahrsagenden Juden ( $v .542 \mathrm{f}$ ) und dem Armenius vel Commagenus haruspex (v. 550) die Chaldaei in einer Weise auf, dass der Name unverkennbar noch eine nationale Nebenbedeutung hat.

Im Folgenden stellen wir nun, ohne auf Vollständigkeit Anspruch zu machen, einige Zeugnisse aus der römischen und griechischen Literatur zusammen, welche uns für die Fortdauer der nationalbabylonischen Sprache und Wissenschaft in Gelehrtenkreisen beweisend zu sein scheinen. Vorausgesetzt ist dabei, dass die Pflege der alteinheimischen Wissenschaft sich fortwährend auf die alten Keilschrifttexte stützte, die jetzt nach vielen Jahrhunderten wieder ans Licht gezogen werden.

Lucret. de rcr. nat. 5, $703 \mathrm{ff.}$ trägt verschiedene Theorien über die Natur des Mondes vor, unter andern die folgende (v. 7 18 ff.):

vcrsarique potest, globus ut sit forte pilai - dimidia ex parti candenti lumine tinctus, versandoque globum variantis edere formas, donique eam partem, quaecumque est ignibus aucta, ad specicm vertit nobis oculosque patentis; inde minutation retro contorquet et aufert luciferam partem glomeraminis atque pilai, ut Babylonica Chaldaeum doctrina, refutans astrologorum artem, contra convincere tendit.

Demnach gab es noch in der ersten Hälfte des ersten Jahrhunderts v. Chr. eine eigene astronomische Schule in Babylon, welche den Theorien sei es der griechischen, sei es (und das ist uns das Wahrscheinlichere, s. sogl.) der konkurrierenden chaldäischen Astronomenschulen (astrologi) gegenüber ihre abweichenden, sicher auf alte Tradition und Literatur zurückgehenden Ansichten festhielt.

Sechs bis acht Jahrzehnte später unterscheidet Strabo 
(I6, I, 6 pag. 739 Cas.) mehrere Astronomenschulen bei den Chaldäern, und zwar bezeichnet er sie deutlich als noch zu seiner Zeit bestehende; zwei derselben, die Schule von Uruk (Arku) und die von Barsip, nennt er mit Namen:

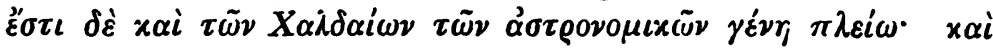

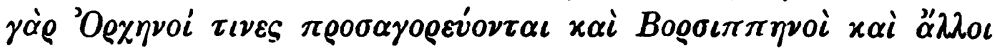

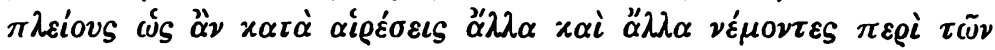
$\alpha \dot{v} \tau\left(\tilde{\nu} \nu \delta c^{\prime} \gamma \mu \alpha \tau \alpha\right.$. In Uruk bestand eine uralte Magierschule $^{1}$, in Barsip ein sehr alter Tempel, der wohl auch wie der von Diodor $(2,9,4)$ erwähnte Belustempel als Sternwarte diente.

Ebenso macht der ältere Plinius (gest. 79 n. Chr.) ausser Babylon selbst zwei chaldäische Hochschulen namhaft, die zu seiner Zeit noch blühten, darunter die altberühmte Doppelstadt Sipar (hist. nat. 6, 26 [30], 123): sunt etiamnum in Mesopotamia oppida Hipparenum ${ }^{2}$ ), Chaldaeorum doctrina et hoc sicut Babylon ... Orcheni quoque, tertia Chaldaeonim doctrina, in eodem situ locantur ad meridiem versi.

Von etwas zweifelhaftem geschichtlichem Wert sind die Angaben des (nach gewöhnlicher Annahme unter Claudius, nach B. G. Niebuhr, welchem RaNKE 3) beipflichtet, erst unter Septimius Severus ${ }^{4}$ ) schreibenden) $Q$. Curtius Rufus, da man nicht immer weiss, aus welchen Quellen er geschöpft und wie weit er diese getreu wiedergegeben

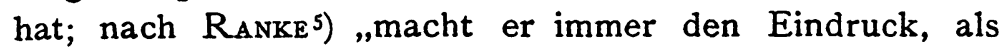
habe er seiner Phantasie dabei vielen Raum gelassen". Immerhin zeigt er sich gerade in babylonischen Dingen

1) Vgl. Lenormant, Die Magie und Wahrsagekunst der Chaldäer, deutsche Ausg. S. 14.

2) Falsche Lesart oder ungeschickte Gräcisierung für Sipparenum. [Vgl. auch Strassmaier bei Epping, Astr. aus Babylon S. 2. - Red.]

3) Weltgeschichte III, $2, \mathrm{~S} .83 \mathrm{f}$.

4) Diese Ansicht Niebuhr's bezeichnet freilich Teuffex (Gesch. der röm. Literatur ${ }^{3} \S 292$, S. 654) als ein Paradoxon.

5) A. a. O. S. 66 . 
ziemlich gut unterrichtet; so wenn er in der Beschreibung des Einzugs Alexanders des Grossen in Babylon Löwen und Panther in Käfigen vorführen lässt (5, I, 2 I), wenn bei dieser festlichen Gelegenheit die nationale Musik nicht fehlen darf (5, 1, 22 vgl. Dan. 3). Merkwürdig ist namentlich die im Wesentlichen mit den Keilschrifttexten ${ }^{\text {) }}$ ) übereinstimmende Andeutung einer Einteilung der Magierkaste in mehrere Klassen. Er schreibt (5, 1, 22): Magi deinde suo more carmen canentes, post hos Chaldaei Babylonionimque non vates modo, sed etiam artifices cum fidibus sui generis ibant. Laudes hi regum canere soliti, Chaldaei siderum motus et statas vices temponım ostendere. Vgl. 3, 3, 9, wo es in der Schilderung der Zugordnung des von Babylon aufbrechenden persischen Heeres heisst: Magi proximi patrium carmen canebant, wo sicherlich nicht an persische, sondern an babylonische Magier zu denken ist.

Curtius unterscheidet also Magi, Chaldaei, Babyloniorum vates und artifices. Die Titulatur ist natürlich unsicher, die Zahl der Klassen mehr oder weniger zufällig und willkürlich, auch ist die Scheidung nicht reinlich durchgeführt; denn was zuerst von den Magi ausgesagt war (suo more carmen canentes), wird hernach den vates und artifices zugeschrieben (laudes hi regum canere soliti). Der Verfasser wirft also wieder zusammen, was er zuvor unterschieden hatte. Das ist aber eben ein Beweis, dass es sich - und das ist in dieser Hinsicht das Wesentliche bei den verschiedenen Bezeichnungen nur um Unterabteilungen einer und derselben Genossenschaft, der Magierkaste, handelt, über deren Organisation, die Unterschiede sowohl als die Zusammengehörigkeit, der Schriftsteller sich nur nicht ganz klar war. Bei all dem aber, was sich für eine genauere Einsicht des Curtius in die babylonischen Zustände und Verhältnisse anführen lässt, bleibt es zweifelhaft, ob wir berechtigt sind, dem, was er über den Ge-

6) LENORMANT a. a. O. S. I5. 
sang der Magier erzählt, den Wert eines geschichtlichen Zeugnisses beizulegen. Diodor, Arrian und Plutarch bieten nichts Entsprechendes. Hat aber Curtius, der auch sonst den Orient ziemlich genau zu kennen scheint, hier aut Grund eigener Anschauung oder guter Information versteht sich : zeitgenössischer - die Berichte seiner Gewährsmänner, die er hier nicht nennt, ausgeschmückt, dann haben wir für unsern $Z$ weck an dem suo more canentes und mehr noch an dem patrium carmen canebant allerdings ein willkommenes Zeugnis - nicht für die Zeit Alexanders des Grossen, für welche es eines solchen gar nicht bedarf, sondern für die römische (vielleicht die spätere römische) Kaiserzeit.

Der am Hof des grossarmenischen Königs Sohämus ( 164 ff. nach Chr.) lebende Literat Jamblichus - nicht der Neuplatoniker! - hat in einen in Babylon spielenden Roman ${ }^{\text {) }}$ Notizen über sich selbst eingeflochten. Diesen zufolge hätte er, ein Vollblutaramäer von Vater- und Mutterseite, bei einem babylonischen Hofmeister, dem er in die Hände fiel, babylonische Sprache, babylonische Lebensart und babylonische Wissenschaft, nämlich Litera-

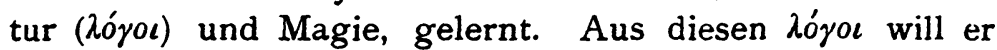
dann seinen Roman geschöpft haben. Letzteres ist natürlich Fiktion, um sein $\delta \rho \alpha \mu \alpha \tau \iota \varkappa o ́ \nu$, wie er's nennt, interessanter zu machen. Im übrigen aber können seine Angaben, soweit sie seine eigene Bildungsgeschichte betreffen, recht wohl zuverlässig sein, d. h. wo nicht Selbsterlebtes, so doch Thatsächliches enthalten. Es heisst in dem von BEKKER mitgeteilten Scholion zum Auszug des Photius:

I) Im Auszug mitgeteilt von Photius, Biblioth. cod. 94, ed. BeKKER I, S. 73 ff. Die autobiographischen Notizen hat aber Photius sehr schlecht excerpiert, zum Teil sehr lückenhaft wiedergegeben, zum Teil gröblich miss-

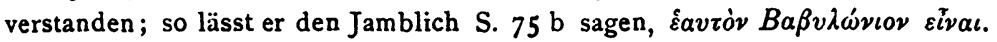
Diesen Mängeln hilft ein Scholion ab, das offenbar aus dem Original den mangelhaiten Auszug des Photius berichtigt und ergänzt (bei BeKKER S. 73, Note zu Zeile 24). Vgl, auch Mommsen, Röm. Giesch. V3, S. 453. 


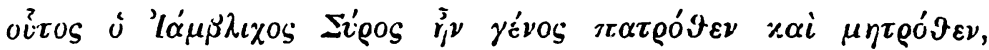

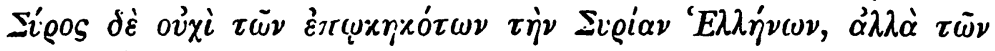

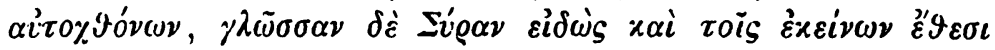

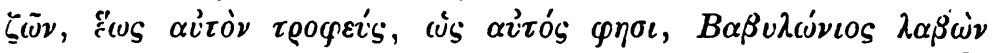

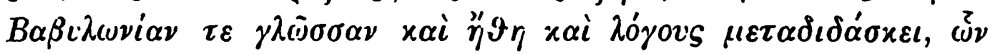

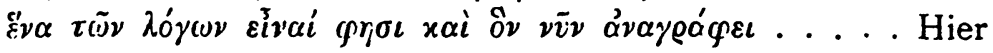
kann nun Photius wieder selbst ergänzend eintreten: $\lambda \dot{\varepsilon} \gamma \varepsilon \iota$ $\delta \dot{\varepsilon} x \alpha i \ldots \mu \mu \alpha \varepsilon \varepsilon \dot{\nu} \tau \dot{\eta} \nu \mu \alpha \gamma \iota x \eta^{\prime} \nu$ (S. 75 b BEKKER). Weiter

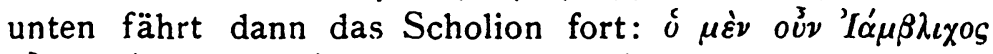

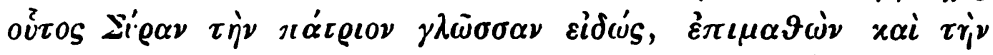

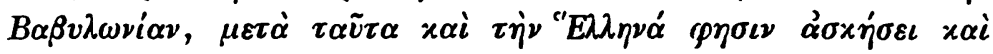
$\chi \varrho \eta \dot{\sigma \varepsilon} \iota \alpha \alpha \beta \varepsilon i v$. Er hat also offenbar sehr bestimmt $z$ wischen der syrischen (aramäischen) und der babylonischen Sprache unterschieden, weiss auch noch von einer in dieser letzteren geschriebenen Literatur, und somit dürfte sich aus seinen Angaben die doppelte Thatsache ergeben:

1) dass noch bis in die Mitte des 2. Jahrhunderts nach Chr. herein der von dem aramäischen Volksidiom, Jamblichs Muttersprache, verschiedene alte babylonische Dialekt als Gelehrtensprache, zum mindesten als Lehrgegenstand, im Gebrauch war, und

2) dass auch die, von der populären aramäischen verschiedene, genuin-babylonische Literatur, und das ist doch wohl die Keilschriftliteratur, in jener Zeit noch gelesen und wissenschaftlich behandelt wurde. 